\title{
Outcomes and Predictors of Mortality in Hospitalized Frail Patients Undergoing Percutaneous Coronary Intervention
}

Rupak Desai ${ }^{1}$, Alok R. Amraotkar ${ }^{2}$, Melissa G. Amraotkar ${ }^{3}$, Samarthkumar Thakkar ${ }^{4}$, Hee Kong Fong ${ }^{5}$, Yash Varma ${ }^{6}$, Nanush Damarlapally ${ }^{7}$, Rajkumar P. Doshi ${ }^{8}$, Kishorbhai Gangani ${ }^{9}$

1. Cardiology, Atlanta Veterans Affairs Medical Center, Decatur, USA 2. Cardiovascular Medicine, University of Louisville School of Medicine, Louisville, USA 3. Nursing Education, University of Louisville School of Nursing, Louisville, USA 4. Internal Medicine, Rochester General Hospital, Rochester, USA 5. Cardiovascular Medicine, University of California Davis Medical Center, Sacramento, USA 6. Internal Medicine, Government Medical College, Bhavnagar, IND 7. Health Sciences, Coleman College of Health Sciences, Houston, USA 8. Internal Medicine, University of Nevada, Reno School of Medicine, Reno, USA 9. Internal Medicine, Texas Health Arlington Memorial Hospital, Arlington, USA

Corresponding author: Alok R. Amraotkar, a.amraotkar@gmail.com

\section{Abstract}

\section{Objective}

To study the impact of frailty on inpatient outcomes among patients undergoing percutaneous coronary intervention (PCI).

\section{Methods}

The National Inpatient Sample data of all PCI-related hospitalizations throughout the United States (US) from 2010 through 2014 was utilized. Patients were divided into two groups: frailty and no-frailty. International Classification of Diseases, Ninth Revision, Clinical Modification (ICD-9-CM) codes were used to stratify groups and outcomes. In order to address the substantial difference in the total number of valid observations between the two groups, a propensity-matched analysis was performed at a 1:1 ratio and caliper width of 0.01 .

\section{Results}

A total of 2,612,661 PCI-related hospitalizations throughout the US from 2010 through 2014 were identified, out of which 16,517 admissions ( $0.6 \%$ ) had coexisting frailty. Only 1:1 propensity-matched data was utilized for the study. Propensity-matched frailty group $(n=14,717)$ as compared to no-frailty $(n=14,755)$ was frequently older, white, and Medicare enrollee $(\mathrm{p}<0.05)$. The frailty group had significantly higher rates of comorbidities and complications ( $\mathrm{p}<0.05$ ). All-cause in-hospital mortality was higher in the no-frailty group $(\mathrm{p}<0.05)$. Age, white race, non-elective admission, urban hospitals, and comorbidities predicted in-hospital

Received 08/05/2019

Review began 08/07/2019 Review ended 08/11/2019 Published 08/16/2019

\section{() Copyright 2019}

Desai et al. This is an open access article distributed under the terms of the Creative Commons Attribution License CC-BY 3.0., which permits unrestricted use, distribution, and reproduction in any medium, provided the original author and source are credited. mortality in frailty group $(\mathrm{p}<0.05)$. Rheumatoid arthritis, depression, hypertension, obesity, dyslipidemia, and history of previous PCI decreased odds of in-hospital mortality in frailty group $(\mathrm{p}<0.05)$. Frailty group had prolonged hospital stay and higher hospital charges $(\mathrm{p}<0.05)$.

\section{Conclusions}

Frailty has a significant effect on PCI-related outcomes. We present a previously unknown protective effect of cardiovascular disease risk factors and other health risk factors on frail patients undergoing PCI. Frailty's inclusion in risk stratification will help in predicting the post-procedure complications and improve resource utilization.

Categories: Cardiology, Family/General Practice, Internal Medicine

Keywords: frailty, all-cause mortality, cardiovascular outcomes

\section{Introduction}

Frailty is described as the presence of three out of the following five criteria of compromised energetics: low grip strength, low energy, slowed walking speed, low physical activity, or unintentional weight loss [1]. Although not a gold standard, there is a broad acceptance of the above criteria in the clinical arena. Frailty significantly affects mortality and disability outcomes [1]. Despite its prognostic importance, frailty is not formally evaluated in clinical practice. Frailty is a known risk factor for cardiovascular diseases (CVD) and their combined deleterious outcomes [2,3]. However, there is limited data on the impact of frailty on CVD outcomes, especially in patients undergoing percutaneous coronary intervention (PCI). PCI is routinely utilized to treat patients with acute or non-acute coronary artery disease effectively, and elderly patients are the most commonly treated demographic population [4]. Given the physical and metabolic changes in elderly 
patients, there is a high chance of encountering frailty in them [5].

The intersection of frailty in cardiac and extra-cardiac comorbidities is critical in understanding the shortand long-term impact of frailty on CVD outcomes. Therefore, we propose a study to investigate the impact of frailty in post-PCI patients. The primary aim of this study is to investigate outcomes (mortality and other complications) and predictors of mortality in hospitalized frail patients undergoing PCI. The second aim of this study is to investigate the impact of frailty on hospital stay.

\section{Materials And Methods \\ Population and design}

The National Inpatient Sample (NIS) is the largest publicly accessible all-payer inpatient database in the United States (US) and is sponsored by the Agency for Healthcare Research and Quality (AHRQ) as a part of the Healthcare Cost and Utilization Project (HCUP) [6]. NIS data from 2010 through 2014 was used for this study. The discharge weights were applied to attain the national estimates, which minimizes the margin of error representing over $95 \%$ of the US population. Adult patients (age 18 and over) undergoing PCI were identified via International Classification of Diseases, Ninth Revision, Clinical Modification (ICD-9-CM) procedural codes $00.66,36.01,36.02,36.05,36.06,36.07$, and 17.55 . Other diagnoses, comorbidities, complications, and discharges were identified using ICD-9-CM codes as detailed in our previous studies. The study population was divided into two groups: "frailty" and a control group - "no frailty". "Frailty” was defined via ICD-9-CM 797, 799.3 \& 783.7 as done previously [7,8]. The study did not require the ethics board approval as NIS is obtainable publicly and does not reveal patients' identifiers.

\section{Primary and secondary outcomes}

There were two primary outcomes in the study. The first primary outcome was defined as inpatient outcomes (mortality, other complications). The second primary outcome was defined as predictors of inhospital mortality (multivariable). The secondary outcome was defined as impact on hospital stay (discharge, length of stay (LOS), and hospital charges). The discharge of patients was further categorized into four sub-categories: routine, transfer to short-term hospital, nursing/other facilities (skilled or intermediate), and other transfers.

\section{Statistical analysis}

A two-tailed $\mathrm{p}<0.05$ was considered a threshold for clinical significance. Due to a substantial difference in the total number of valid observations between the two groups, a propensity-matched analysis was performed with a ratio of $1: 1$ without replacement using a caliper width of 0.01 . The absolute standardized difference of $<10 \%$ was obtained for most variables before and after propensity matching (Figure 1 ). 
Standardized Differences Before Matching

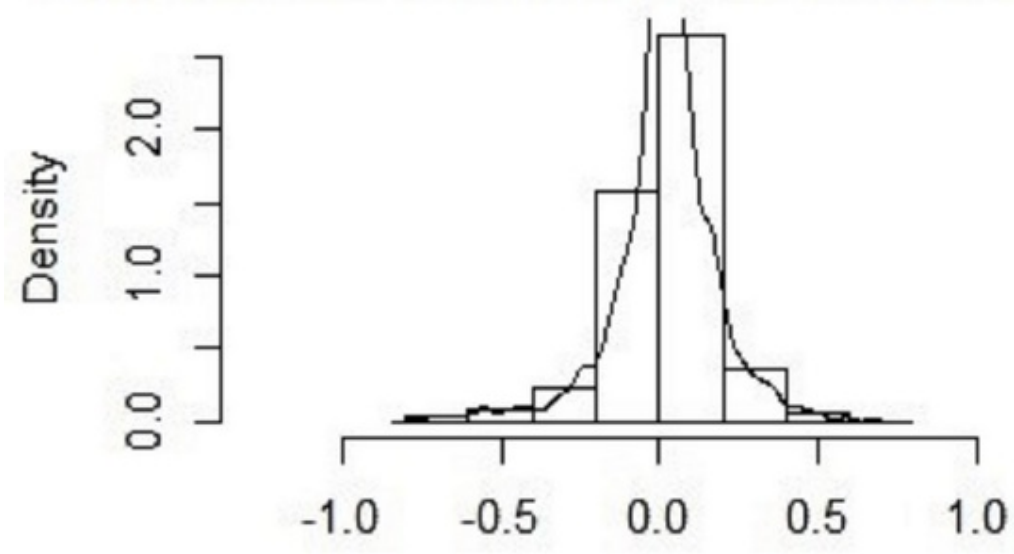

Std. difference

\section{Standardized Differences After Matching}

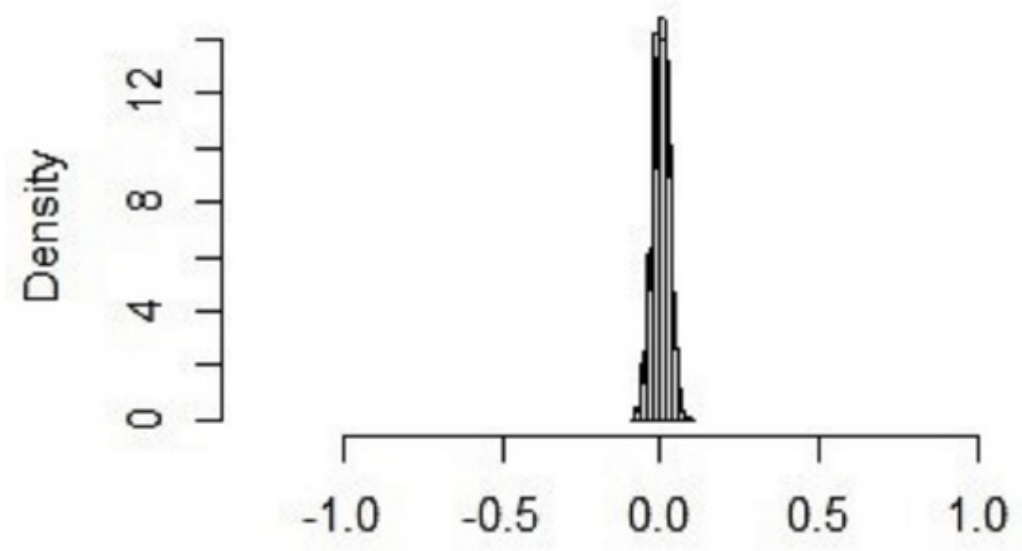

Std. difference

FIGURE 1: Standardized Differences in Data Before and After 1:1 Propensity Matching

Std. Difference $=$ Standardized Differences

Data was matched with all baseline characteristics, comorbidities, and hospital characteristics. Only 1:1 propensity-matched data were utilized to assess primary and secondary outcomes. Chi-square test and independent-sample t-test were performed to compare the baseline characteristics. Outcomes and predictors were adjusted for age, sex, race, median income, payer status, hospital characteristics, and relevant comorbidities. Odds ratios (OR) and 95\% confidence intervals (CI) were calculated for mortality predictors. International Business Machines Corporation (IBM) Statistical Package for the Social Sciences (SPSS) v22.0 (IBM Corp., Armonk, NY) was utilized to perform the analyses.

\section{Results}




\section{Cureus}

\section{General cohort characteristics}

A total of 2,612,661 PCI-related hospitalizations throughout the US from 2010 through 2014 were identified, out of which 16,517 admissions $(0.6 \%$ ) had coexisting frailty (Tables 1,2$)$. Only 1:1 propensity-matched data was utilized for this study. The unmatched original data are detailed in Tables 1,2. After a 1:1 propensitymatching, there were 14,717 patients in the frailty group and 14,755 patients in the no-frailty group (Tables $3,4)$.

\begin{tabular}{|c|c|c|c|}
\hline Characteristics & No-Frailty $(n=2,596,144)$ & Frailty $(n=16,517)$ & p-value \\
\hline Age Mean \pm SD (Yrs) & $64 \pm 12$ & $75 \pm 11$ & $<0.001$ \\
\hline Sex & & & $<0.001$ \\
\hline Male (\%) & 67.1 & 49.5 & \\
\hline Female (\%) & 32.9 & 50.5 & \\
\hline Race & & & $<0.001$ \\
\hline White (\%) & 76.7 & 78.3 & \\
\hline African American (\%) & 9.2 & 9.5 & \\
\hline Hispanic (\%) & 7.3 & 7.3 & \\
\hline Asian Pacific Islander (\%) & 2.4 & 2.0 & \\
\hline Native American (\%) & 0.6 & 0.4 & \\
\hline Others & 3.7 & 2.5 & \\
\hline Type Of Admission & & & $<0.001$ \\
\hline Non-Elective (\%) & 82.2 & 90.0 & \\
\hline Elective (\%) & 17.8 & 10.0 & \\
\hline Admission Day & & & $<0.001$ \\
\hline Weekday (\%) & 79.6 & 76.8 & \\
\hline Weekend (\%) & 20.4 & 23.2 & \\
\hline Median Household Income ${ }^{*}$ & & & $<0.001$ \\
\hline $0-25^{\text {th }}(\%)$ & 28.7 & 32.9 & \\
\hline $26-50^{\text {th }}(\%)$ & 27.0 & 28.2 & \\
\hline $51-75^{\text {th }}(\%)$ & 24.4 & 22.4 & \\
\hline $76-100^{\text {th }}(\%)$ & 19.9 & 16.4 & \\
\hline Primary Expected Payer & & & $<0.001$ \\
\hline Medicare (\%) & 51.4 & 80.7 & \\
\hline Medicaid (\%) & 7.2 & 4.3 & \\
\hline Private (\%) & 31.6 & 11.8 & \\
\hline Self-Pay/No Charge/Other (\%) & 9.9 & 3.2 & \\
\hline Location/Teaching Status Of Hospital & & & $<0.001$ \\
\hline Rural (\%) & 6.4 & 6.9 & \\
\hline Urban Non-Teaching (\%) & 37.3 & 40.8 & \\
\hline Urban Teaching (\%) & 56.3 & 52.3 & \\
\hline Region Of Hospital & & & $<0.001$ \\
\hline Northeast (\%) & 18.5 & 3.9 & \\
\hline
\end{tabular}




\section{Cureus}

\begin{tabular}{|lcc|}
\hline Midwest (\%) & 25.3 & 32.1 \\
\hline South (\%) & 39.3 & 48.7 \\
West (\%) & 16.9 & 15.3
\end{tabular}

TABLE 1: General Characteristics of No-Frailty versus Frailty Patients Undergoing Percutaneous Coronary Intervention Without Any Propensity Matching

*Derived from https://www.hcup-us.ahrq.gov/db/vars/zipinc_qrtl/nisnote.jsp

$\mathrm{SD}=$ Standard Deviation

p-value $<0.05$ indicates statistical significance

\begin{tabular}{|c|c|c|c|}
\hline Characteristics & No-Frailty $(n=2,596,144)$ & Frailty $(n=16,517)$ & p-value \\
\hline Alcohol Abuse (\%) & 2.6 & 2.9 & 0.012 \\
\hline Chronic Pulmonary Disease (\%) & 17.3 & 33.0 & $<0.001$ \\
\hline Coagulopathy (\%) & 3.2 & 8.6 & $<0.001$ \\
\hline Congestive Heart Failure (\%) & 1.3 & 6.9 & $<0.001$ \\
\hline Depression (\%) & 6.9 & 12.7 & $<0.001$ \\
\hline Hypertension (\%) & 73.6 & 76.6 & $<0.001$ \\
\hline Diabetes, With Chronic Complications (\%) & 5.3 & 11.7 & $<0.001$ \\
\hline Diabetes, Uncomplicated (\%) & 31.2 & 34.1 & $<0.001$ \\
\hline Dyslipidemia (\%) & 70.7 & 62.2 & $<0.001$ \\
\hline Drug Abuse (\%) & 2.0 & 1.6 & 0.002 \\
\hline Fluid And Electrolyte Disorders (\%) & 13.2 & 41.9 & $<0.001$ \\
\hline Valvular Heart Disease (\%) & 0.4 & 2.1 & $<0.001$ \\
\hline Obesity (\%) & 15.6 & 17.8 & $<0.001$ \\
\hline Peripheral Vascular Disorders (\%) & 11.1 & 20.2 & $<0.001$ \\
\hline Renal Failure (\%) & 13.7 & 33.7 & $<0.001$ \\
\hline Smoking (\%) & 41.8 & 31.1 & $<0.001$ \\
\hline
\end{tabular}

TABLE 2: Comorbidities in No-Frailty versus Frailty Patients Undergoing Percutaneous Coronary Intervention Without Any Propensity Matching

p-value $<0.05$ indicates statistical significance

\begin{tabular}{|c|c|c|c|}
\hline Characteristics & No Frailty $(n=14,755)$ & Frailty $(n=14,717)$ & p-value \\
\hline Age Mean $\pm S D$ (Years) & $73 \pm 11$ & $75 \pm 11$ & $<0.001$ \\
\hline Sex & & & 0.99 \\
\hline Male (\%) & 49.6 & 49.6 & \\
\hline Female (\%) & 50.4 & 50.4 & \\
\hline Race & & & $<0.001$ \\
\hline
\end{tabular}




\section{Cureus}

\begin{tabular}{|c|c|c|c|}
\hline White (\%) & 79.5 & 78.9 & \\
\hline African American (\%) & 8.9 & 8.8 & \\
\hline Hispanic (\%) & 7.1 & 7.5 & \\
\hline Asian Pacific Islander (\%) & 1.9 & 2.0 & \\
\hline Native American (\%) & 0.1 & 0.4 & \\
\hline Others & 2.3 & 2.4 & \\
\hline Type Of Admission & & & 0.83 \\
\hline Non-Elective (\%) & 90.0 & 89.9 & \\
\hline Elective (\%) & 10.0 & 10.1 & \\
\hline Admission Day & & & 0.10 \\
\hline Weekday (\%) & 77.9 & 77.1 & \\
\hline Weekend (\%) & 22.1 & 22.9 & \\
\hline Median Household Income* & & & 0.54 \\
\hline $0-25^{\text {th }}(\%)$ & 33.6 & 32.9 & \\
\hline $26-50^{\text {th }}(\%)$ & 27.3 & 27.9 & \\
\hline $51-75^{\text {th }}(\%)$ & 22.4 & 22.5 & \\
\hline $76-100^{\text {th }}(\%)$ & 16.8 & 16.7 & \\
\hline Primary Expected Payer & & & $<0.001$ \\
\hline Medicare (\%) & 82.3 & 80.7 & \\
\hline Medicaid (\%) & 3.9 & 4.3 & \\
\hline Private (\%) & 11.6 & 11.8 & \\
\hline Self-Pay/No Charge/Other (\%) & 2.3 & 3.2 & \\
\hline Location/Teaching Status Of Hospital & & & 0.47 \\
\hline Rural (\%) & 6.3 & 6.6 & \\
\hline Urban Non-Teaching (\%) & 42.5 & 42.1 & \\
\hline Urban Teaching (\%) & 51.3 & 51.3 & \\
\hline Region Of Hospital & & & 0.09 \\
\hline Northeast (\%) & 3.8 & 4.2 & \\
\hline Midwest (\%) & 27.4 & 27.9 & \\
\hline South (\%) & 52.5 & 52.0 & \\
\hline West (\%) & 16.4 & 15.9 & \\
\hline
\end{tabular}

TABLE 3: General Characteristics of No-Frailty versus Frailty Patients Undergoing Percutaneous Coronary Intervention From 1:1 Propensity Matched Data

*Derived from https://www.hcup-us.ahrq.gov/db/vars/zipinc_qrtl/nisnote.jsp

$\mathrm{SD}=$ Standard Deviation

p-value $<0.05$ indicates statistical significance 


\section{Cureus}

\begin{tabular}{|c|c|c|c|}
\hline Comorbidities & No Frailty $(n=14,755)$ & Frailty $(n=14,717)$ & p-value \\
\hline Alcohol Abuse (\%) & 2.8 & 3.0 & 0.51 \\
\hline Chronic Pulmonary Disease (\%) & 32.7 & 32.4 & 0.61 \\
\hline Coagulopathy (\%) & 7.9 & 8.5 & 0.041 \\
\hline Congestive Heart Failure (\%) & 5.8 & 7.0 & $<0.001$ \\
\hline Depression (\%) & 12.2 & 12.6 & 0.33 \\
\hline Hypertension (\%) & 76.4 & 76.4 & 0.98 \\
\hline Diabetes, With Chronic Complications (\%) & 12.4 & 11.8 & 0.65 \\
\hline Diabetes, Uncomplicated (\%) & 33.8 & 34.1 & 0.12 \\
\hline Dyslipidemia (\%) & 63.4 & 62.2 & 0.037 \\
\hline Drug Abuse (\%) & 1.9 & 1.7 & 0.21 \\
\hline Fluid And Electrolyte Disorders (\%) & 41.3 & 42.4 & 0.07 \\
\hline Valvular Heart Disease (\%) & 1.5 & 2.3 & $<0.001$ \\
\hline Obesity (\%) & 18.2 & 17.9 & 0.50 \\
\hline Peripheral Vascular Disorders (\%) & 19.6 & 20.2 & 0.23 \\
\hline Renal Failure (\%) & 34.0 & 33.7 & 0.57 \\
\hline Smoking (\%) & 30.7 & 31.2 & 0.34 \\
\hline
\end{tabular}

TABLE 4: Comorbidities in No-Frailty versus Frailty Patients Undergoing Percutaneous Coronary Intervention From 1:1 Propensity Matched Data

p-value $<0.05$ indicates statistical significance

\section{Propensity matched cohort characteristics}

General cohort characteristics of 1:1 propensity-matched cohort are presented in Table 3. The frailty group was frequently older (mean age $75 \pm 11$ years), white ( $78.9 \%)$, and Medicare enrollees ( $80.7 \%)$. Cohort comorbidities are presented in Table 4 . The frailty group had significantly higher rates of coagulopathy $(8.5 \%$ vs $7.9 \%, \mathrm{p}=0.041)$, congestive heart failure ( $7 \%$ vs $5.8 \%, \mathrm{p}<0.001)$, and valvular heart disease $(2.3 \%$ vs $1.5 \%$, $\mathrm{p}<0.001$ ) as compared to no-frailty group (Table 2 ). However, dyslipidemia was lower in the frailty group (62.2\% vs. $63.4 \%, \mathrm{p}=0.037$ ) as compared to the no-frailty group (Table 4 ).

First primary outcome: inpatient outcomes in no-frailty and frailty groups

The propensity-matched inpatient outcomes (mortality and other complications) are presented in Figure 2. The all-cause inpatient mortality was significantly higher in no-frailty group ( $5 \%$ vs $4.3 \%$ ). Postoperative myocardial infarction ( $15.3 \%$ vs $11.3 \%$ ), pericardial complications ( $1.4 \%$ vs $0.8 \%$ ), iatrogenic cardiac complications ( $2.8 \%$ vs $2 \%$ ), postoperative hypotension/shock ( $0.9 \%$ vs $0.5 \%$ ), postoperative respiratory failure ( $2.3 \%$ vs $1.6 \%$ ), pneumo-hemothorax ( $1.5 \%$ vs $1.1 \%$ ), postoperative infection ( $7.5 \%$ vs $4.2 \%$ ), and acute kidney injury during dialysis $(7.7 \%$ vs $6.7 \%)$ were all significantly higher in the frailty group (all p $<0.05)$ (Figure 2). 


\section{Cureus}

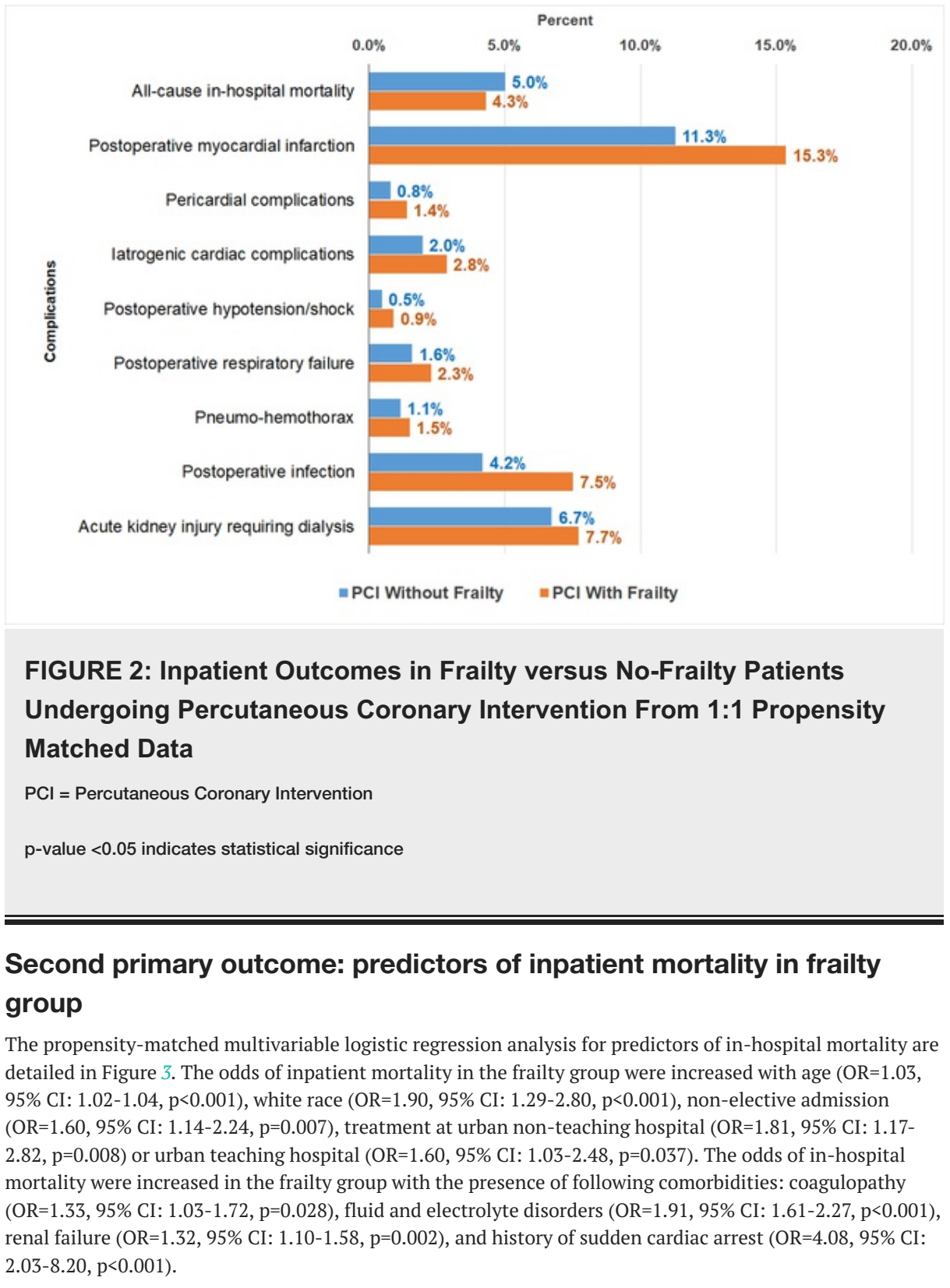



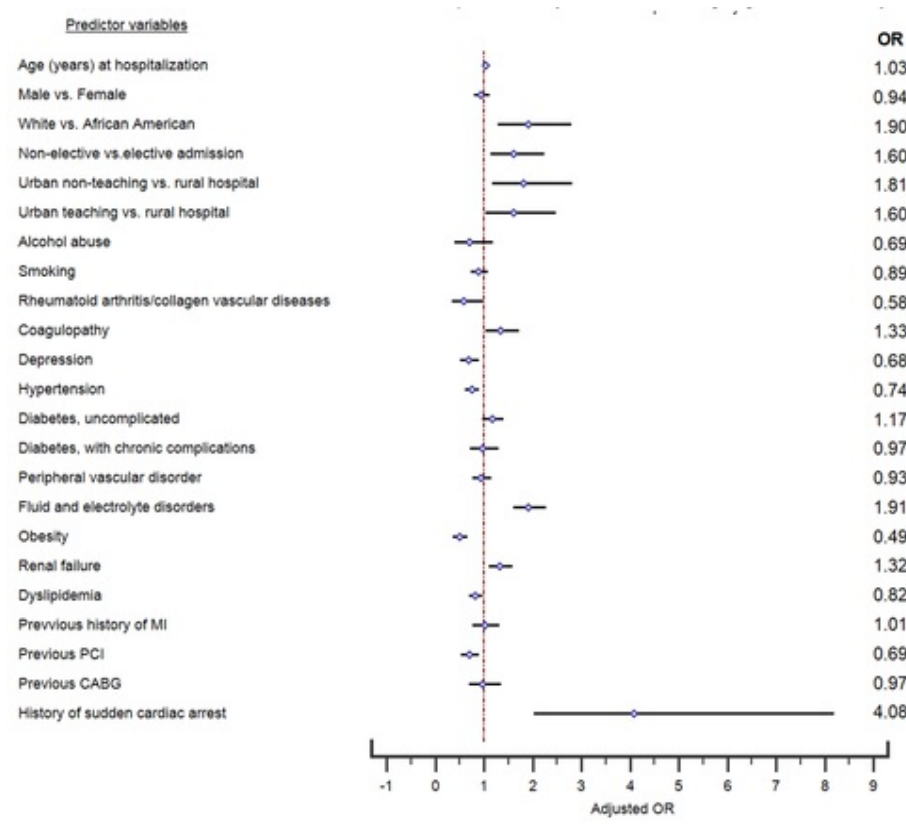

$95 \% \mathrm{Cl}$

1.02-1.04

0.80-1.12

1.29-2.80

$1.14-2.24$

$1.17-2.82$

1.03-2.48

$0.40-1.19$

$0.73-1.09$

$0.35-0.98$

1. $03-1.72$

$0.51-0.90$

$0.62 \cdot 0.90$

$0.97-1.41$

$0.71-1.30$

$0.76-1.15$

$1.61-2.27$

$0.36-0.66$

1.10-1.58

0.69-0.97

$0.77-1.32$

$0.53-0.90$

0.69-1.36

2.03-8.20

FIGURE 3: Multivariable Predictors of Inpatient Mortality in Frailty Patients From 1:1 Propensity Matched Data

$\mathrm{CABG}=$ Coronary Artery Bypass Graft, $\mathrm{Cl}=$ Confidence Interval, $\mathrm{OR}=$ Odds Ratio, $\mathrm{MI}=$ Myocardial Infarction, $\mathrm{P}=\mathrm{p}$-value, $\mathrm{PCl}=$ Percutaneous Coronary Intervention

p-value $<0.05$ indicates statistical significance

The odds of in-hospital mortality were decreased in the frailty group with the presence of following comorbidities: rheumatoid arthritis/collagen vascular diseases (OR=0.58, 95\% CI: 0.35-0.98, $p=0.043$ ), depression ( $\mathrm{OR}=0.68,95 \% \mathrm{CI}: 0.51-0.90, \mathrm{p}=0.007)$, hypertension ( $\mathrm{OR}=0.74,95 \% \mathrm{CI}: 0.62-0.90, \mathrm{p}=0.002)$, obesity (OR=0.49, 95\% CI: 0.36-0.66, $\mathrm{p}<0.001$ ), dyslipidemia ( $\mathrm{OR}=0.82,95 \% \mathrm{CI}: 0.69-0.97, \mathrm{p}=0.023$ ), and history of previous PCI (OR=0.69, 95\% CI: 0.53-0.90, $\mathrm{p}=0.007)$.

\section{Secondary outcome: impact on hospital stay (discharge, LOS, and hospital charges)}

Routine discharges $(65.2 \%$ vs $30.2 \%$, $\mathrm{p}<0.001)$ and transfers to short-term hospital $(1.4 \%$ vs $0.9 \%$, $\mathrm{p}<0.001)$ were significantly higher in the no-frailty group. Whereas, transfers to nursing/other facilities ( $21.9 \%$ vs. $14 \%, \mathrm{p}<0.001)$ and all other transfers $(42.2 \%$ vs. $14.3 \%$, $\mathrm{p}<0.001)$ were significantly higher in the frailty group. Frailty group had prolonged mean LOS $(9.5 \pm 7.8$ vs $5.9 \pm 6.6$ days, $p<0.001)$ and higher mean hospital charges $(\$ 141,532$ vs $\$ 108,468, \mathrm{p}<0.001)$.

\section{Discussion}

In this retrospective analysis of PCI-related hospitalizations in frail and non-frail patients, we provide a first-ever attempt at quantifying the impact of frailty on PCIs performed across the US between 2010 and 2014. There were three major novel findings with important clinical implications in this study. First, with the exception of all-cause in-hospital mortality, a majority of in-hospital complications were significantly associated with frailty in patients undergoing PCI. Second, there was a > $50 \%$ chance of in-hospital mortality in frail patients if they were-white, non-electively admitted, treated at an urban hospital, had

fluid/electrolyte disorders, or a history of sudden cardiac arrest. A history of sudden cardiac arrest alone increased the chance of in-hospital mortality in frail patients by $>300 \%$. Third, the chances of in-hospital mortality in frail patients decreased by $>20 \%$ if patients had coexisting rheumatoid arthritis/collagen vascular diseases, depression, hypertension, obesity, dyslipidemia, or a history of previous PCI. Additionally, the cost of treatment and length of stay were both significantly higher in frail patients as compared to nonfrail patients.

There is a high incidence of post-procedural complications in older patients undergoing cardiovascular interventions, which may be due to a higher number of comorbidities, recurrent multivessel diseases, and or disparities in resource utilization [9-12]. Moreover, frailty reduces the abilities of stress management, thereby accentuating patients' suffering [13]. In our study, the post-PCI all-cause in-hospital mortality rate was higher in non-frail patients than frail patients, which is contradictory to the findings of previous studies 
[9-11]. Our study presents a novel finding that a history of previous PCI decreased the odds of mortality in frail patients, thereby highlighting a protective effect of a history of PCI for this population. One of the reasons for such contradictory findings could be decreased CVD burden at the time of the study, secondary to successful disease management in the preceding PCIs.

In stark contrast with expected clinical outcomes, pre-existing rheumatoid arthritis, depression, hypertension, obesity, and dyslipidemia a were all associated with reduced odds of inpatient mortality in frail patients undergoing PCI. These findings may have considerably influenced the observed decrease in overall in-hospital mortality in frail patients in our study. Consistent with prior reports of the "Obesity Paradox", frail patients with obesity in our study were protected (by almost 50\%) against post-PCI inhospital mortality. The protective effect of obesity in critically ill patients has been revealed in multiple recent studies [14,15]. In a recently published study, Leistner et al. found abnormally low BMI in elderly patients associated with increased mortality [15]. Similarly, both Lavie et al. (2014) and Kapoor et al. (2010), have found an association of frailty and cachexia with poorer prognosis as compared to being obese in patients with heart failure $[14,16]$. The neutralization of inflammatory signals by adipose tissue via the production of soluble receptors may be a key factor in this phenomenon [17]. Lipid-lowering treatment has been previously shown not to affect mortality in the elderly population, and low total cholesterol is in-fact associated with higher mortality [18]. This can explain the protective effect of dyslipidemia on in-hospital mortality in frail patients in our study.

Although smoking remains a widely reported CVD risk factor for worse outcomes, it did not play a role in predicting short-term outcomes for frail patients in our study. The rising burden of endocrine disorders, including diabetes mellitus and hypothyroidism among the US population, is known to play a role in PCI outcomes [19]. However, in our study, we did not observe a correlation between diabetes or hypothyroidism and in-hospital negative outcomes in frail patients.

Between varieties of different hospital admission causes, the black race has historically been associated with lower resource utilization and higher in-hospital mortality [10-12,20-22]. However, among frail patients in our study, the white race was the strongest racial predictor of inpatient mortality. This finding could be due to the sociodemographic profile of the study sample with a majority of the cohort (with or without frailty) being white.

CVD is the leading cause of death in the US [23]. Paradoxically, hypertension, obesity, and dyslipidemia, the three major CVD risk factors, were found to decrease the odds of in-hospital mortality in frail patients in our study. These findings warrant further investigations regarding the role of these CVD risk factors in predicting short-term or long-term outcomes in frail patients undergoing revascularization.

\section{Limitations}

As with any administrative database study, this study has a few potential limitations. Under/over-reporting of frailty could be a limitation considering our methodology involved ICD-9-CM codes to extract frail patients with a possibility of administrative coding errors. The database does not provide follow-up data. In addition, we could not grade the severity of frailty through indices.

\section{Conclusions}

Frailty has a significant effect on PCI-related outcomes and should be included as a fundamental clinical condition while evaluating risk factors for inpatient outcomes and mortality benefits surrounding PCI. This study presents a previously unknown protective effect of CVD risk factors and other health risk factors on frail patients undergoing PCI procedure. Specifically designed studies are warranted to further investigate the paradoxical findings of expected clinical outcomes in this study, which will add vital value for managing this growing patient population.

\section{Additional Information}

\section{Disclosures}

Human subjects: Consent was obtained by all participants in this study. Animal subjects: All authors have confirmed that this study did not involve animal subjects or tissue. Conflicts of interest: In compliance with the ICMJE uniform disclosure form, all authors declare the following: Payment/services info: All authors have declared that no financial support was received from any organization for the submitted work. Financial relationships: All authors have declared that they have no financial relationships at present or within the previous three years with any organizations that might have an interest in the submitted work. Other relationships: All authors have declared that there are no other relationships or activities that could appear to have influenced the submitted work.

\section{Acknowledgements}

SHARED FIRST AUTHORSHIP: R. Desai and A.R. Amraotkar contributed equally to this manuscript and should be considered co-first authors. AUTHOR CONTRIBUTIONS: Conception and Design: R. Desai, A.R. 
Amraotkar Collection and assembly of Data: R. Desai, A.R. Amraotkar Drafting of Article: All authors Critical Revision for Important Intellectual Content: All authors

\section{References}

1. Fried LP, Tangen CM, Walston J, et al.: Frailty in older adults: evidence for a phenotype . J Gerontol A Biol Sci Med Sci. 2001, 56:146-156. 10.1093/gerona/56.3.M146

2. Matsuzawa Y, Konishi M, Akiyama E, et al.: Association between gait speed as a measure of frailty and risk of cardiovascular events after myocardial infarction. J Am Coll Cardiol. 2013, 61:1964-1972.

10.1016/j.jacc.2013.02.020

3. Veronese N, Cereda E, Stubbs B, et al.: Risk of cardiovascular disease morbidity and mortality in frail and pre-frail older adults: results from a meta-analysis and exploratory meta-regression analysis. Ageing Res Rev. 2017, 35:63-73. 10.1016/j.arr.2017.01.003

4. Endorsed by the Latin American Society of Interventional Cardiology, PCI Writing Committee, Levine GN, et al.: 2015 ACC/AHA/SCAI focused update on primary percutaneous coronary intervention for patients with ST-elevation myocardial infarction: an update of the 2011 ACCF/AHA/SCAI guideline for percutaneous coronary intervention and the 2013 ACCF/AHA guideline for the management of ST-elevation myocardial infarction: a report of the American College of Cardiology/American Heart Association Task Force on Clinical Practice Guidelines and the Society for Cardiovascular Angiography and Interventions. Catheter Cardiovasc Interv. 2016, 87:1001-1019. 10.1002/ccd.26325

5. Hansson GK, Hermansson A: The immune system in atherosclerosis . Nat Immunol. 2011, 12:204-212. 10.1038/ni.2001

6. Healthcare cost and utilization project (HCUP) database overview of the national (nationwide) inpatient sample. (2019). Accessed: July 2019: https://www.hcup-us.ahrq.gov/nisoverview.jsp.

7. Ahmed AK, Goodwin CR, De la Garza-Ramos R, Kim RC, Abu-Bonsrah N, Xu R, Sciubba DM: Predicting short-term outcome after surgery for primary spinal tumors based on patient frailty. World Neurosurg. 2017, 108:393-398. 10.1016/j.wneu.2017.09.034

8. Anzaldi LJ, Davison A, Boyd CM, Leff B, Kharrazi H: Comparing clinician descriptions of frailty and geriatric syndromes using electronic health records: a retrospective cohort study. BMC Geriatr. 2017, 17:248. 10.1186/s12877-017-0645-7

9. Gharacholou SM, Roger VL, Lennon RJ, Rihal CS, Sloan JA, Spertus JA, Singh M: Comparison of frail patients versus nonfrail patients $>/=65$ years of age undergoing percutaneous coronary intervention. Am J Cardiol. 2012, 109:1569-1575. 10.1016/j.amjcard.2012.01.384

10. Desai R, Mirza O, Sachdeva R, Kumar G: Sex and racial disparities in fractional flow reserve-guided percutaneous coronary intervention utilization: a 5-year national experience. Ann Transl Med. 2018, 6:198. 10.21037/atm.2018.03.15

11. Desai R, Patel U, Fong HK, et al.: Modern-day nationwide utilization of intravascular ultrasound and its impact on the outcomes of percutaneous coronary intervention with coronary atherectomy in the United States. J Ultrasound Med. 2019, Epub ahead of print. 10.1002/jum.14922

12. Desai R, Singh S, Fong HK, et al.: Racial and sex disparities in resource utilization and outcomes of multivessel percutaneous coronary interventions (a 5-year nationwide evaluation in the United States). Cardiovasc Diagn Ther. 2019, 9:18-29. 10.21037/cdt.2018.09.02

13. Lee DH, Buth KJ, Martin BJ, Yip AM, Hirsch GM: Frail patients are at increased risk for mortality and prolonged institutional care after cardiac surgery. Circulation. 2010, 121:973-978. 10.1161/circulationaha.108.841437

14. Lavie CJ, De Schutter A, Alpert MA, Mehra MR, Milani RV, Ventura HO: Obesity paradox, cachexia, frailty, and heart failure. Heart Fail Clin. 2014, 10:319-326. 10.1016/j.hfc.2013.12.002

15. Leistner DM, Bazara S, Münch C, et al.: Association of the body mass index with outcomes in elderly patients ( $\geqslant 80$ years) undergoing percutaneous coronary intervention. Int J Cardiol. 2019, 292:73-77. 10.1016/j.ijcard.2019.06.044

16. Kapoor JR, Heidenreich PA: Obesity and survival in patients with heart failure and preserved systolic function: a U-shaped relationship. Am Heart J. 2010, 159:75-80. 10.1016/j.ahj.2009.10.026

17. Mohamed-Ali V, Goodrick S, Bulmer K, Holly JM, Yudkin JS, Coppack SW: Production of soluble tumor necrosis factor receptors by human subcutaneous adipose tissue in vivo. Am J Physiol. 1999, 277:971-975. 10.1152/ajpendo.1999.277.6.E971

18. Petersen LK, Christensen K, Kragstrup J: Lipid-lowering treatment to the end? A review of observational studies and RCTs on cholesterol and mortality in 80+-year olds. Age Ageing. 2010, 39:674-680. 10.1093/ageing/afq129

19. Desai R, Singh S, Syed MH, et al.: Temporal trends in the prevalence of diabetes decompensation (diabetic ketoacidosis and hyperosmolar hyperglycemic state) among adult patients hospitalized with diabetes mellitus: a nationwide analysis stratified by age, gender, and race. Cureus. 2019, 11:4353. 10.7759/cureus.4353

20. Becker ER, Rahimi A: Disparities in race/ethnicity and gender in in-hospital mortality rates for coronary artery bypass surgery patients. J Natl Med Assoc. 2006, 98:1729-1739.

21. Jones JM, Fingar KR, Miller MA, et al.: Racial disparities in sepsis-related in-hospital mortality: using a broad case capture method and multivariate controls for clinical and hospital variables, 2004-2013. Crit Care Med. 2017, 45:1209-1217. 10.1097/CCM.0000000000002699

22. Nguyen GC, Laveist TA, Segev DL, Thuluvath PI: Race is a predictor of in-hospital mortality after cholecystectomy, especially in those with portal hypertension. Clin Gastroenterol Hepatol. 2008, 6:11461154. 10.1016/j.cgh.2008.05.024

23. Naghavi M, Abajobir AA, Abbafati C, et al.: Global, regional, and national age-sex specific mortality for 264 causes of death, 1980-2016: a systematic analysis for the Global Burden of Disease Study 2016. Lancet. 2017, 390:1151-1210. 10.1016/S0140-6736(17)32152-9 